\title{
CRYSTAL FIELD THEORY OF MAGNETIC BEHAVIOUR OF $\mathrm{Er}_{2}\left(\mathrm{SO}_{4}\right)_{3} \cdot 8 \mathrm{H}_{2} \mathrm{O}$
}

\author{
R. Gupta, U.S. Ghosh and C. Basu \\ Department of Solid State Physics, Indian Association for the Cultivation of Science \\ Jadavpur, Calcutta - 700032, India
}

(Received September 19, 1995; revised version February 7, 1996)

\begin{abstract}
Using the most rigorous approach of direct diagonalization of the IIamiltonian matrix constructed in the complete basis states (364 in number) of the $\mathrm{Er}^{3+}$ ion the observed magnetic susceptibility and anisotropy data of erbium sulphate octahydrate were simulated and the set of crystal field parameters was evaluated. This procedure automatically includes the effect of intermediate coupling and the $J$-mixing under the crystal field. It was also demonstrated that such rigorous calculation using complete basis states is necessary in order to evaluate the correct set of parameters which may widely differ from the values obtained from approximate calculations previously performed using partial number of basis states of the ion concerned.
\end{abstract}

PACS numbers: $71.70 . \mathrm{Ch}, 75.10 . \mathrm{Dg}$

\section{Introduction}

The present paper reports the results of simulation of experimental magnetic susceptibility and anisotropy data of Er-sulphate octahydrate by using wave functions which were computed from crystal field theory in the most rigorous way. The crystal field (CF) parameters obtained during the process are considered to be accurate ones. For accurate evaluation of any parameter, firstly it is necessary to evaluate it in a way consistent with all the physical quantities which involve it. Secondly, the most rigorous procedure of calculation of those physical quantities should be followed. Various physical quantities of a rare earth (RE) ion like optical energy levels, EPR $g$-values, magnetic susceptibility and anisotropy values involve the crystal ficld. The most rigorous procedure of calculating these quantities is the direct diagonalization of the complete IIamiltonian matrix constructed in a basis of states belonging to all the atomic terms of the rare earth ion concerned. This procedure automatically includes intermediate coupling and different $J$-mixing under the crystal field. Although this rigorous approach has been adopted for interpreting optical absorption data of rare earth ions by a number of workers [1-7] the magnetic data of ions are usually interpreted in terms of a simpler theory, not rigorous 
from the above point of view. In a recent communication [8] we demonstrated in the case of magnetic susceptibility of $\operatorname{Pr}_{2}\left(\mathrm{SO}_{4}\right)_{3} \cdot 8 \mathrm{H}_{2} \mathrm{O}$ that there is wide difference between the crystal field parameters obtained by a simpler method and that obtained by rigorous calculation and we have concluded that this rigorous procedure is necessary for the analysis of magnetic data. Unfortunately, for Er-sulphate octahydrate optical data and magnetic resonance data are lacking. In the absence of these data we followed the rigorous procedure and evaluated the correct crystal field parameters from the analysis of magnetic susceptibility and anisotropy data alone. It is to be noted that for $\mathrm{Er}^{3+}$ ion the total matrix spans 364-dimensional space. Previous workers $[9,10]$ confined their calculation within 52-dimensional space resulting from the states of multiplets ${ }^{4} I_{15 / 2},{ }^{4} I_{13 / 2},{ }^{4} I_{11 / 2}$ and ${ }^{4} I_{9 / 2}$ of the ground term ${ }^{4} I$ of $\mathrm{Er}^{3+}$ ion. But one has to work in 364-dimensional space for correct evaluation of crystal field parameters and indeed this has been done in the present paper.

\section{Theoretical consideration}

\subsection{Crystal field energy levels}

The model Hamiltonian of $\mathrm{Er}^{3+}$ ion in the crystal in the absence of external field is written as

$$
H=H_{\mathrm{r}}^{i j}+H_{\mathrm{so}}+H_{\mathrm{cf}},
$$

where $H_{\mathbf{r}}^{i j}$ is the electrostatic energy, $H_{\text {so }}$ is the spin-orbit interaction energy and $H_{\text {cf }}$ represents the crystal field interaction. There are other interactions which we do not consider. They are two-body configuration, three-body configuration, spin-other-orbit and electrostatically correlated spin-orbit interaction etc. For detailed calculation of energy levels these interactions may become necessary. Since optical data are lacking in this case, and only the low lying group of Stark levels contribute to the magnetic susceptibility we did not consider these interactions. One of our objectives is to demonstrate how the results differ when one works with 364-dimensional space rather than 52-dimensional one. Since previous workers considered the interactions given by Hamiltonian in Eq. (1) we also consider the same for the sake of comparison.

The rare earth sulphate single crystal form an isomorphous series [11]. Following the work of Sherry [12] the $\mathrm{RE}^{3+}$ ion in the octahydrated sulphates is coordinated by eight oxygen atoms of which four come from the $\mathrm{SO}_{4}^{2-}$ and four from $\mathrm{H}_{2} \mathrm{O}$ arranged in the form of a distorted Archimedes antiprism. Therefore the site symmetry of the $\mathrm{RE}^{3+}$ ion in these crystals may be taken as tetragonal to a first approximation. Then $H_{\mathrm{cf}}$ is of the form

$$
H_{\mathrm{cf}}=\sum_{i}-|e| V(i)=\sum_{i} \sum_{k, q}-|e| a_{k q} U_{q}^{(k)},
$$

where $k=2,4,6, q(|q| \leq k)=0, \pm 4, a_{k q}$ is the coefficient associated with the spherical harmonics $Y_{k}^{q}$ when the crystal field potential $V$ is expanded in terms of them and $a_{k q}=a_{k-q}$ for the site symmetry of the ion in our case. In (2) $U_{q}^{(k)}=$ $r^{k} Y_{k}^{q}$ is an irreducible tensor operator and the summation over $i$ extends over the $4 f$ electrons. Quantities like $-|e| a_{k q}{ }_{4 f}\left\langle r^{k}\right\rangle_{4 f}$ will occur in the various matrix 
elements of the $\mathrm{CF}$ interaction and they are treated as crystal field parameters denoted by $B_{k q}$.

The matrix of the Hamiltonian $H$ is constructed in a basis of states represented by $\left|S L J M_{J}\right\rangle$ considering all the terms of $\mathrm{Er}^{3+}$ ion and it comes out to be a $364 \times 364$ matrix. Upon diagonalization a low-lying group consisting of eight Kramers doublets (i.e. sixteen states in all) with a spread of $\approx 218 \mathrm{~cm}^{-1}$ is obtained. The next higher group of levels is about $6000 \mathrm{~cm}^{-1}$ above the ground group of Stark levels. These low-lying eight Kramers doublets are responsible for the significant contribution to the susceptibility, the higher levels give negligible contribution.

\subsection{Calculation of the paramagnetic susceptibility}

As mentioned earlier, the low-lying eight Kramers doublets resulting from the diagonalization of the energy matrix are to be considered for the calculation of magnetic susceptibility. Therefore we now apply Zeeman perturbation on eight Kramers doublets only. The ionic paramagnetic susceptibility along and perpendicular to the symmetry axis at different temperatures was worked out using Van Vleck's formula [13]

$$
K_{\alpha}=N \sum\left[\frac{\left(W_{\alpha}^{(1)}\right)^{2}}{k_{\mathrm{B}} T}-2 W_{\alpha}^{(2)}\right] \exp \left(-\frac{W^{(0)}}{k_{\mathrm{B}} T}\right) / \sum \exp \left(-\frac{W^{(0)}}{k_{\mathrm{B}} T}\right),
$$

where the summation extends over all the lowest sixteen states comprising the eight Kramers doublets. $W^{(0)}$ is the unperturbed energy of the state in the zero magnetic field (i.e. crystal field energy), $W_{\alpha}^{(1)} H$ and $W_{\alpha}^{(2)} H^{2}$ are the first- and second-order energy correction of the state due to Zeeman perturbation $\beta H\left(L_{\alpha}+2 S_{\alpha}\right)$ when the magnetic field $H$ is applied along the direction $\alpha$.

\subsection{Calculation of $g$-values}

For the calculation of the principal $g$-values one is interested only in the lowest doublet $\left(\phi_{1}, \phi_{1}^{\prime}\right)$ (say) in the crystal field level pattern. The expressions for the $g$-values are

$$
\begin{aligned}
& g_{\|}=g_{z}=\left|\left\langle\phi_{1}\left|L_{z}+2 S_{z}\right| \phi_{1}\right\rangle-\left\langle\phi_{1}^{\prime}\left|L_{z}+2 S_{z}\right| \phi_{1}^{\prime}\right\rangle\right|, \\
& g_{\perp}=g_{x}=g_{y}=2\left|\left\langle\phi_{1}\left|L_{x}+2 S_{x}\right| \phi_{1}^{\prime}\right\rangle\right|=2\left|\left\langle\phi_{1}\left|L_{y}+2 S_{y}\right| \phi_{1}^{\prime}\right\rangle\right| .
\end{aligned}
$$

\section{Results and discussion}

The $364 \times 364$ matrix formed from the states $\left|S L J M_{J}\right\rangle$ of all the atomic terms of $\mathrm{Er}^{3+}$ ion is diagonalized. It breaks up into four matrices of order 91 each under a crystal field of $D_{4 h}$ symmetry. $\mathrm{Er}^{3+}$ being an odd electron ion, the ground state is a Kramers doublet. As stated earlier, a low-lying group of sixteen states comprising eight Kramers doublets occurs with an overall energy separation of $\approx 218 \mathrm{~cm}^{-1}$. The energy values of these states along with their crystal quantum. number $\mu$ are given in Table. 


\section{TABLE}

Calculated energy values along with the crystal quantum number $\mu$ of the ground Stark level.

\begin{tabular}{c|c}
\hline \hline$\mu$ & Energy in $\mathrm{cm}^{-1}$ \\
\hline $\pm 1 / 2$ & 0 \\
$\pm 1 / 2$ & 13.60 \\
$\pm 1 / 2$ & 25.44 \\
$\pm 3 / 2$ & 51.93 \\
$\pm 3 / 2$ & 131.64 \\
$\pm 3 / 2$ & 144.29 \\
$\pm 1 / 2$ & 201.79 \\
$\pm 3 / 2$ & 218.97
\end{tabular}

With these sixteen crystal field states we calculate the mean magnetic susceptibility $\bar{K}$ and the principal magnetic susceptibilities $K_{\|}$and $K_{\perp}$ of the $\mathrm{Er}^{3+}$ ion in Er-sulphate octahydrate at different temperatures from $300 \mathrm{~K}$ to $80 \mathrm{~K}$ and try to fit the calculated result with the experimental values $[9,14]$. To start with we used the parameters given by previous workers [10] in our rigorous procedure and obtained the mean susceptibility $\bar{K}$ and the principal ionic susceptibilities $K_{\|}$ and $K_{\perp}$. It is observed that with the parameters quoted by previous workers the difference between the theoretical and experimental values throughout the temperature range from room to liquid nitrogen varies from $11.25-13.7 \%, 26.7-39 \%$ and $0.14-5.2 \%$ for $\bar{K}, K_{\|}$and $K_{\perp}$ respectively. For $\bar{K}$ the agreement is not so good, for $K_{\|}$the agreement is very poor, for $K_{\perp}$ the agreement is good, no doubt. But there is serious disagreement between observed and calculated values of magnetic anisotropy. From observed magnetic anisotropy values $K_{\|}>K_{\perp}$. But here calculated $K_{\perp}$ becomes larger than $K_{\|}$. Therefore we conclude that with these parameters overall agreement is not satisfactory. Next we systematically varied the parameters and best possible fit is obtained with the following set of parameters:

$$
\begin{array}{lll}
E^{1}=6558.88 \mathrm{~cm}^{-1}, & E^{2}=32.70 \mathrm{~cm}^{-1}, & E^{3}=631.83 \mathrm{~cm}^{-1}, \\
\zeta=2393 \mathrm{~cm}^{-1}, & B_{20}=208.9 \mathrm{~cm}^{-1}, & B_{40}=-84.54 \mathrm{~cm}^{-1}, \\
B_{60}=650.28 \mathrm{~cm}^{-1}, & B_{44}=157.14 \mathrm{~cm}^{-1}, & B_{64}=-29.25 \mathrm{~cm}^{-1},
\end{array}
$$

where $E^{1}, E^{2}, E^{3}$ are electrostatic energy parameters which are linear combinations of Slater $F^{k}$ integrals introduced by Racah [15], $\zeta$ is the spin-orbit coupling coefficient, $B_{k q}$ 's are crystal field parameters. The ranges of deviation of calculated values from experiment for $\bar{K}, K_{\|}$and $K_{\perp}$ are $6.9-8.5 \%, 5-9 \%$ and $5-10 \%$ respectively and also $K_{\|}>K_{\perp}$ throughout the range of temperature studied in agreement with experimental observation. The observed thermal variation of $\bar{K}$, $K_{\|}$and $K_{\perp}$ are shown in Figs. 1, 2 and 3 along with our theoretically computed curves. Each figure also includes the curve computed by using the parameters of 


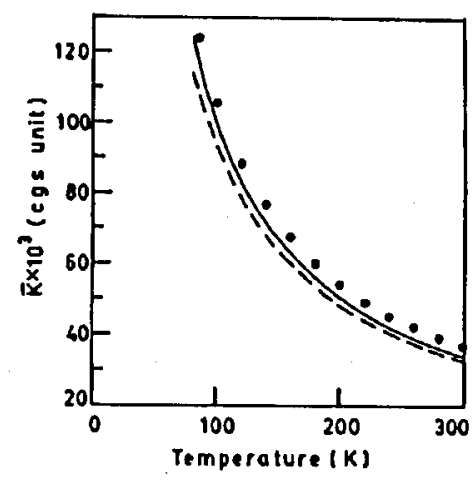

Fig. 1. Thermal variation of $\bar{K}$ for Er-sulphate octahydrate. - theoretical curve (calculated with our parameters), - - - theoretical curve (calculated with previous workers' parameters), • experimental points.

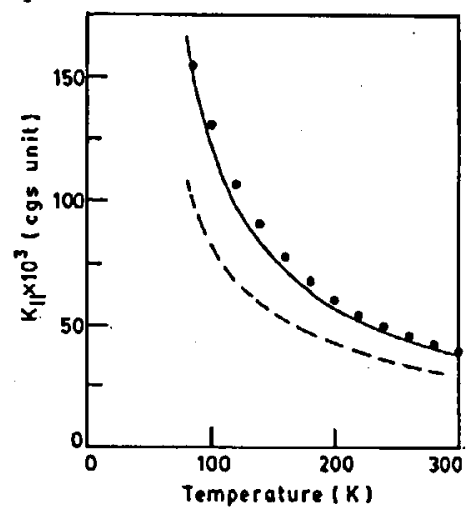

Fig. 2. Thermal variation of $K_{\|}$for Er-sulphate octahydrate. - theoretical curve (calculated with our parameters), - - - theoretical curve (calculated with previous workers' parameters), - experimental points.

previous workers for comparison. Using the wave functions of the lowest Kramers doublet in Eq. (4) we also calculated the EPR $g$-values which come out to be $g_{\|}=8.24, g_{\perp}=0.42$ against 12.36 and 1.0 respectively as calculated by previous workers [10]. However, in absence of the experimental data the calculated $g$-values could not be compared with the experiment.

It may appear that with our set of parameters the limit of agreement (a maximum deviation of $10 \%$ between the experimental and calculated magnetic susceptibilities) is still not very satisfactory though the agreement is considered to be much improved than that of the previous workers. But it could be seen that if experimental values at one temperature are fitted well, the values at other temperatures diverge to a great extent. Results have been fitted in such a way that for all the three quantities $\bar{K}, K_{\|}$and $K_{\perp}$ the deviation between theoretical and experimental values is small and somewhat similar. The disagreement that 


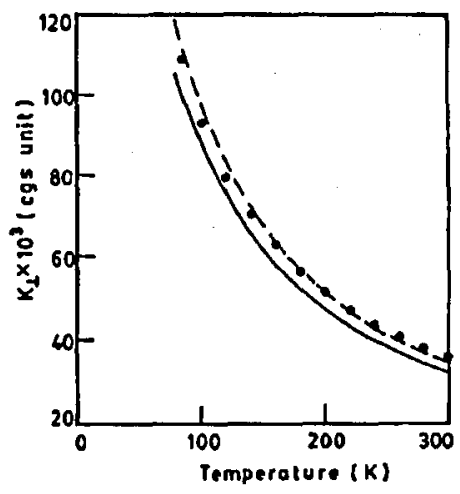

Fig. 3. Thermal variation of $K_{\perp}$ for Er-sulphate octahydrate. - theoretical curve (calculated with our parameters), - - theoretical curve (calculated with previous workers' parameters), • experimental points.

still persists between theoretical and experimental values may be due to the fact that actual symmetry may be slightly different from $D_{4 h}$.

It appears to us that the quality of fit obtained is quite indicative of the correctness of the parameter values. In all we have nine parameters (three related to electrostatic interaction, one to spin-orbit interaction and five to crystal field). It is found that in the case of rare earth ions electrostatic parameters and the spin-orbit parameter in different crystals differ very little from their corresponding free ion values. The present set of values of these parameters for $\mathrm{Er}_{2}\left(\mathrm{SO}_{4}\right)_{3} \cdot 8 \mathrm{H}_{2} \mathrm{O}$ compares quite satisfactorily with the free ion values. Therefore, we are finally left with five free parameters which are adjusted to reproduce twenty four data points, twelve for each of the magnetic susceptibility and the magnetic anisotropy experiments. However, each of it would have been better if we could fit spectral data, EPR $g$-values and magnetic data simultaneously. But in the absence of spectral and EPR data we have predicted the $g$-values and one can easily evaluate the spectral data by using the parameter values which may be compared with the experimental data when available.

Finally we stress that the major emphasis of the present investigation is to demonstrate that one has to use the complete basis in contrast to the partial basis as is customary with magnetic workers [16-21] and diagonalize the energy matrix incorporating all the interactions simultaneously. This is necessary to get the correct set of parameters as has already been shown by us for other system [8]. It has been clearly demonstrated by our calculations shown in Figs. 1-3 that the present method (with complete basis set) gives distinct improvement in agreement with experiment using the same number of parameters that has been used earlier. Such an improved agreement is not possible to achieve with a truncated basis set. The truncated basis set calculation even predicts the wrong sign of the anisotropy of susceptibility. It is to be noted that the values of parameters evaluated by us differ much from those of previous workers. Our set of parameters is considered to be correct one. In conclusion we emphasize that for the analysis of magnetic data 
the full matrix, rather than the partial matrix constructed from states belonging to the ground term only, should be considered.

\section{References}

[1] J.B. Gruber, J. Chem. Phys. 38, 946 (1963).

[2] J.C. Eisenstein, J. Chem. Phys. 39, 2134 (1963).

[3] J. Stöhr, J.B. Gruber, Chem. Phys. 7, 336 (1975).

[4] C.K. Jayasankar, F.S. Richardson, Phys. Status Solidi B 155, 221 (1989).

[5] C. Görller-Walrand, K. Binnemans, L. Fluyt, J. Phys. Condens. Matter 5, 8359 (1993).

[6] E. Rukmini, C.K. Jayasankar, M.F. Reid, J. Phys. Condens. Matter 6, 5919 (1994).

[7] C. Görller-Walrand, E. Huygen, K. Binnemans, L. Fluyt, J. Phys. Condens. Matter 6, 7797 (1994).

[8] R. Gupta, C. Basu, U.S. Ghosh, Indian J. Phys. A 69, 603 (1995).

[9] D. Neogy, A. Mookerji, J. Phys. Soc. Japan 20, 1332 (1965).

[10] D. Neogy, A.K. Mukherjee, T. Purohit, J. Phys. Chem. Solids 47, 919 (1986).

[11] W.H. Zachariasen, J. Chem. Phys. 3, 197 (1935).

[12] E.G. Sherry, J. Solid State Chem. 19, 271 (1976).

[13] J.H. Van Vleck, Electric and Magnetic Susceptibilities, Oxford University Press, London 1932.

[14] L.C. Jackson, Proc. Phys. Soc. (Great Britain) 47, 1027 (1935).

[15] G. Racah, Phys. Rev. 76, 1352 (1949).

[16] D. Neogy, T. Purohit, J. Magn. Magn. Mater. 68, 344 (1987).

[17] D. Neogy, T. Purohit, Phys. Rev. B 35, 5849 (1987).

[18] A. Sengupta, S. Bhattacharyya, D. Ghosh, Phys. Lett. A 140, 261 (1989).

[19] T. Purohit, D. Neogy, R.K. Saha, J. Magn. Magn. Mater. 117, 399 (1992).

[20] A. Sengupta, D. Ghosh, B.M. Wanklyn, Phys. Rev. B 47, 8281 (1993).

[21] A. Sengupta, D. Ghosh, J. Phys. Chem. Solids 56, 35 (1995). 